\title{
From symmetric spaces to buildings, curve complexes and outer spaces
}

\author{
Lizhen $\mathrm{Ji}^{*}$
}

\begin{abstract}
In this article, we explain how spherical Tits buildings arise naturally and play a basic role in studying many questions about symmetric spaces and arithmetic groups, why Bruhat-Tits Euclidean buildings are needed for studying S-arithmetic groups, and how analogous simplicial complexes arise in other contexts and serve purposes similar to those of buildings.

We emphasize the close relationships between the following: (1) the spherical Tits building $\Delta_{\mathbb{Q}}(\mathbf{G})$ of a semisimple linear algebraic group $\mathbf{G}$ defined over $\mathbb{Q},(2)$ a parametrization by the simplices of $\Delta_{\mathbb{Q}}(\mathbf{G})$ of the boundary components of the Borel-Serre partial compactification $\bar{X}^{\mathrm{BS}}$ of the symmetric space $X$ associated with $\mathbf{G}$, which gives the Borel-Serre compactification of the quotient of $X$ by every arithmetic subgroup $\Gamma$ of $\mathbf{G}(\mathbb{Q})$, (3) and a realization of $\bar{X}^{\mathrm{BS}}$ by a truncated submanifold $X_{T}$ of $X$. We then explain similar results for the curve complex $\mathcal{C}(S)$ of a surface $S$, Teichmüller spaces $T_{g}$, truncated submanifolds $T_{g}(\varepsilon)$, and mapping class groups $\operatorname{Mod}_{g}$ of surfaces. Finally, we recall the outer automorphism groups $\operatorname{Out}\left(F_{n}\right)$ of free groups $F_{n}$ and the outer spaces $X_{n}$, construct truncated outer spaces $X_{n}(\varepsilon)$, and introduce an infinite simplicial complex, called the core graph complex and denoted by $\mathcal{C G}\left(F_{n}\right)$, and we then parametrize boundary components of the truncated outer space $X_{n}(\varepsilon)$ by the simplices of the core graph complex $\mathcal{C G}\left(F_{n}\right)$. This latter result suggests that the core graph complex is a proper analogue of the spherical Tits building.

The ubiquity of such relationships between simplicial complexes and structures at infinity of natural spaces sheds a different kind of light on the importance of Tits buildings.
\end{abstract}

Keywords: Tits building, symmetric space, curve complex, outer space, arithmetic group, mapping class group, outer automorphism group of free group

MSC 2000: 20E42, 32G15, 20E06,14L30

*Partially supported by NSF grant DMS-0604878 


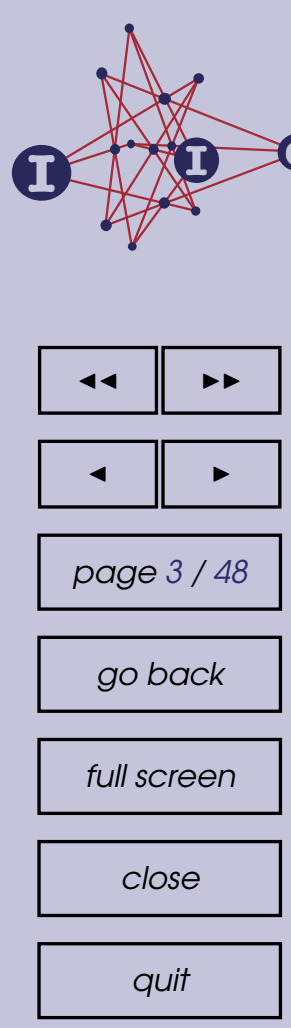

$\Delta_{\mathbb{Q}}(\mathbf{G})$ of an algebraic group $\mathbf{G}$ defined over $\mathbb{Q}[11]$. We also explain that the Solomon-Tits theorem on the homotopy type of $\Delta_{\mathbb{Q}}(\mathbf{G})$ can be used to show that arithmetic subgroups $\Gamma$ are virtual duality groups, but not virtual Poincaré duality groups if $\Gamma \backslash X$ are not compact [11]. We discuss a realization of the partial compactification $\bar{X}^{\mathrm{BS}}$ by a truncated submanifold $X_{T}$ of $X[40,35]$, which is convenient for many applications and generalizations. Furthermore, we use the Borel-Serre compactification $\overline{\Gamma \backslash X}^{\mathrm{BS}}$ and its relationship to the Tits building $\Delta_{\mathbb{Q}}(\mathbf{G})$ to determine completely the ends of locally symmetric spaces $\Gamma \backslash X$ (Proposition 4.7).

S-arithmetic subgroups such as $\mathrm{GL}\left(n, \mathbb{Z}\left[\frac{1}{p_{1}}, \ldots, \frac{1}{p_{k}}\right]\right)$, where $p_{1}, \ldots, p_{k}$ are prime numbers, are natural generalizations of arithmetic subgroups such as $\mathrm{GL}(n, \mathbb{Z})$. We explain how Bruhat-Tits buildings are naturally needed in order to study S-arithmetic subgroups. A Bruhat-Tits building can be compactified by adding a corresponding spherical Tits building, and this compactification was used to prove that $\mathrm{S}$-arithmetic subgroups of semisimple linear algebraic groups are duality groups [12].

More importantly, we also try to explain and emphasize in this article the following basic point, in a variety of settings, that natural simplicial complexes can be used to understand geometry and structures at infinity of noncompact spaces, as in the above case of buildings for symmetric spaces and locally symmetric spaces.

One setting is that of the Teichmüller space $T_{g}$ associated with a closed, orientable surface $S=S_{g}$ of genus $g \geq 2$. Its structure at infinity, in particular the boundary of a Borel-Serre type partial compactificattion ${\overline{T_{g}}}^{\text {BS }}$ can be described in terms of an infinite simplicial complex, called the curve complex and denoted by $\mathcal{C}(S)$ [21]. Together with an analogue of the Solomon-Tits Theorem for the curve complex $\mathcal{C}(S)$, it can be shown the mapping class group $\operatorname{Mod}_{g}$ is a virtual duality group [20] but not a virtual Poincaré duality group [24]. The partial compactification ${\overline{T_{g}}}^{\text {BS }}$ can also be realized by a truncated submanifold $T_{g}(\varepsilon)$, whose boundary components can be easily seen to be parametrized by the simplices of the curve complex $\mathcal{C}(S)$.

Another setting is that of the outer automorphism group $\operatorname{Out}\left(F_{n}\right)$ of a free group $F_{n}$ on $n$-generators. It acts properly on the associated outer space $X_{n}$ of marked metric graphs with fundamental group equal to $F_{n}$ [17]. In order to prove that $\operatorname{Out}\left(F_{n}\right)$ is a virtual duality group, a Borel-Serre type partial compactification $\bar{X}_{n}$ BS of $X_{n}$ was constructed [8]. An infinite simplicial complex, called the free factor complex and which is similar to a Tits building was constructed for $F_{n}$, and an analogue of the Solomon-Tits theorem was also proved in [22]. On the other hand, the expected relationship between the free factor 


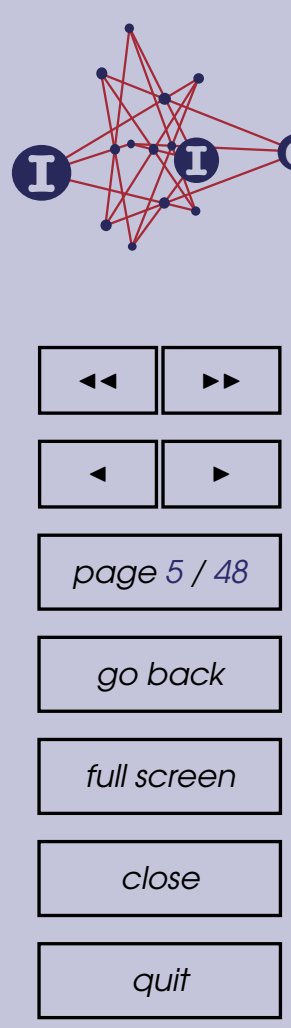

of the spherical Tits building $\Delta(G)$.

By definition, a complete Riemannian manifold $M$ is called a symmetric space if for every point $x \in M$, the geodesic symmetry $s_{x}$, which reverses every geodesic passing through $x$, is defined locally and gives a local diffeomorphism of $M$ at $x$, is a global isometry of $M$.

Let $G$ be the identity component of the isometry group $\operatorname{Isom}(M)$ of $M$. Then $G$ acts transitively on $X$. For any base point $x \in X$, denote the stabilizer of $x$ in $G$ by $K$. Then $X$ can be identified with the homogeneous space $G / K$, and the Riemannian metric on $X$ is equal to a $G$-invariant metric induced from an inner product on the tangent space $T_{x} X$.

The Euclidean spaces $\mathbb{R}^{n}$ with the standard metric and their quotients by discrete isometry groups are symmetric spaces. Besides these flat symmetric spaces, irreducible symmetric spaces are classified into two types: compact and non-compact types.

In terms of curvature, a symmetric space of compact type has nonnegative sectional curvature and strictly positive Ricci curvature, and is compact. Furthermore, its fundamental group is finite.

On the other hand, a symmetric space of non-compact type has nonpositive sectional curvature and strictly negative Ricci curvature. It is also simply connected. Hence it is a so-called Hadamard manifold, and in particular it is diffeomorphic to its tangent space $T_{x} X$ (more precisely, the exponential map $\exp _{x}: T_{x} X \rightarrow X$ is a diffeomorphism.)

In this paper, we are mainly concerned with symmetric spaces of noncompact type. In terms of the identification $X=G / K$ above, the Lie group $G=\operatorname{Isom}(X)^{0}$ is a connected semisimple noncompact Lie group with trivial center, and the stabilizer $K$ is a maximal compact subgroup of $G$.

Conversely, for any semisimple Lie group $G$ with finitely many connected components and finite center, let $K$ be a maximal compact subgroup of $G$, which is unique up to conjugation by elements of $G$. Then the homogeneous space $X=G / K$ with a $G$-invariant metric is a Riemannian symmetric space of noncompact type.

The main example of symmetric spaces for us arises as follows. Let $\mathbf{G} \subset$ $\mathrm{GL}(n, \mathbb{C})$ be a semisimple linear algebraic group defined over $\mathbb{Q}$. Then the real locus $G=\mathbf{G}(\mathbb{R})$ is a real semisimple Lie group with finitely many connected components and finite center. Note that even if $\mathbf{G}$ is connected, $G$ is not necessarily connected.

Besides symmetric spaces $X$ of noncompact type, we will also study their quotients $\Gamma \backslash X$ by discrete isometry groups $\Gamma$. We will concentrate on the case that $\Gamma \backslash X$ have finite volume with respect to the measure induced from the 


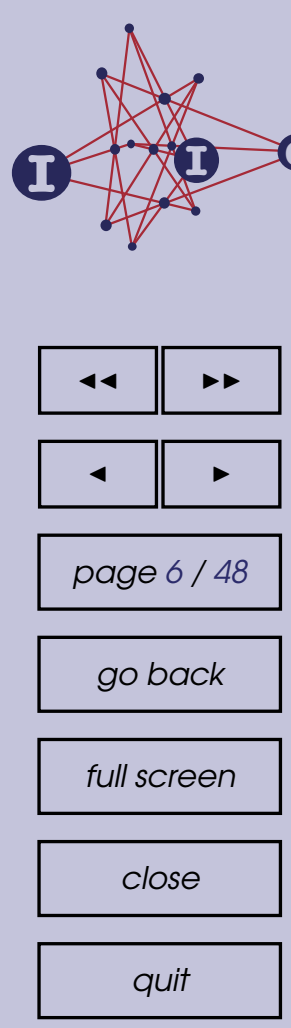

invariant metric. In terms of the identification $X=G / K, \Gamma$ is a discrete subgroup of $G$ such that the quotient $\Gamma \backslash G$ has finite volume with respect to any Haar measure of $G$, i.e., $\Gamma$ is a lattice subgroup of $G$. If $G=\mathbf{G}(\mathbb{R})$ is the real locus of $\mathbb{Q}$-algebraic group as above, then an important class of lattice subgroups consists of arithmetic subgroups of $\mathbf{G}(\mathbb{Q})$.

Besides the above connections with Lie groups and algebraic groups, symmetric spaces $X$ and locally symmetric spaces $\Gamma \backslash X$ are important in other fields such as harmonic analysis, differential geometry, algebraic geometry, number theory, and rigidity theory. For example, the Poincaré upper half plane $\mathbb{H}^{2}$, i.e., the simply connected surface with constant negative sectional curvature -1 , is the symmetric space associated with $G=\mathrm{SL}(2, \mathbb{R})$ and $K=\mathrm{SO}(2)$, and the quotient $S L(2, \mathbb{Z}) \backslash \mathbb{H}^{2}$ is the moduli space of all elliptic curves.

Understanding the geometry of $X$ has led to many important notions, and relationships between the geometry of $X$ and group theoretic structures of $G$ have played an important role in many problems. One important notion is the notion of maximal flat subspaces, often called maximal flats or simply flats in the following. By definition, a maximal flat subspace of $X$ is a maximal totally geodesic flat subspace. It is known that all maximal flats of $X$ are isometric to each other, and their common dimension is called the rank of $X$. Since every geodesic in $X$ is a totally geodesic flat subspace of dimension 1 , the rank of $X$ is at least 1 .

If $X=G / K$ and $G=\mathbf{G}(\mathbb{R})$ is the real locus of a semisimple linear algebraic group defined over $\mathbb{Q}$ and hence over $\mathbb{R}$, then the rank of $X$ is equal to the $\mathbb{R}$-rank of $\mathbf{G}$ as an algebraic group.

\section{The geodesic compactification}

Since $X$ is non-compact, a natural problem is to understand its geometry at infinity. One way to understand its geometry at infinity is to study its compactifications and their relationship to its geometry. One such compactification is the geodesic compactification $X \cup X(\infty)$ which is obtained by adding as the boundary the set of equivalence classes of directed geodesics.

More specifically, two unit speed directed geodesics $\gamma_{1}(t), \gamma_{2}(t)$ of $X$ are called equivalent if

$$
\limsup _{t \rightarrow+\infty} d\left(\gamma_{1}(t), \gamma_{2}(t)\right)<+\infty
$$

Denote the equivalence class containing a geodesic $\gamma$ by $[\gamma]$, and the set of all equivalence classes of unit speed geodesics in $X$ by $X(\infty)$.

Since $X$ is simply connected and nonpositively curved, it can be shown that for any base point $x \in X$, every equivalence class of geodesics contains exactly 




\section{The Dirichlet problem on $X \cup X(\infty)$}

The construction of the geodesic compactification $X \cup X(\infty)$ works for any Hadamard manifold. An important problem for Hadamard manifolds is the solvability of the Dirichlet problem on $X \cup X(\infty)$ : given a continuous function $f$ on $X(\infty)$, find a harmonic function $u$ on $X$ with boundary value equal to $f$, i.e., $u$ can be extended continuously to the boundary $X(\infty)$ and $\left.u\right|_{X(\infty)}=f$.

When the Hadamard manifold is given by a simply connected Riemannian manifold with sectional curvature pinched by two negative numbers, this Dirichlet problem is solvable (see [1] and the references therein). For more general rank 1 Hadamard manifolds which admit compact quotients, the Dirichlet problem is also solvable [3] (see also [5] for the identification of the Poisson boundary of $X$ with $X(\infty)$ ). (Note that in this case, the rank of a Hadamard manifold $M$ is defined to the minimum of the dimension of vector spaces of parallel Jacobi fields along geodesics in $M$.)

On the other hand, if $X$ is a symmetric space of noncompact type and of rank at least 2 , then the above Dirichlet problem is not solvable. The basic reason is that the Poisson boundary of $X$ can be identified with a proper subset of $X(\infty)$. For example, consider $X=\mathbb{H}^{2} \times \mathbb{H}^{2}$, where $\mathbb{H}^{2}$ is the Poincaré upper half plane. Then the rank of $X$ is equal to 2 . Suppose that the Dirichlet problem is solvable on $X \cup X(\infty)$. Then, for every continuous function $f$ on $X(\infty)$, we obtain a bounded harmonic function $u_{f}$ on $X$ with the boundary value $f$. Now every bounded harmonic function $u_{f}(x, y)$ on the product $\mathbb{H}^{2} \times \mathbb{H}^{2}$ splits as a product $u_{1}(x) u_{2}(y)$, where $u_{1}, u_{2}$ are bounded harmonic functions on $\mathbb{H}^{2}$. (Note that this splitting follows from the result that the Poisson boundary of $X$ is given by the distinguished boundary $\mathbb{H}^{2}(\infty) \times \mathbb{H}^{2}(\infty)$. See [18] for references on the Poisson boundary of symmetric spaces of noncompact type.) If a sequence $x_{j}$ goes to a boundary point in $\mathbb{H}^{2}(\infty)$ but $y$ is fixed, then $\left(x_{j}, y\right)$ will converge to a boundary point in $X(\infty)$ independent of $y$. Then the assumption that the boundary value of $u_{f}(x, y)=u_{1}(x) u_{2}(y)$ is equal to $f$ implies that $u_{2}(y)$ must be independent of $y$ if $\lim _{j \rightarrow+\infty} u_{1}\left(x_{j}\right) \neq 0$. Similarly, we can conclude that if a sequence $y_{j}$ goes to a boundary point and $\lim _{j \rightarrow+\infty} u_{2}\left(y_{j}\right) \neq 0$, then $u_{1}(x)$ is constant. This implies that $u(x, y)$ is a constant function. This clearly contradicts the condition that the boundary value of $u_{f}$ is equal to $f$ if $f$ is not constant. Therefore, in this case, the Dirichlet problem is not solvable for any non-constant continuous function $f$ on $X(\infty)$.

It is perhaps also worthwhile to point out that it is still an open problem if a general Hadamard manifold of higher rank that is not a product is a symmetric space, though the answer is positive if it admits a finite volume quotient. See $[41,2]$ for summaries and references of this rank rigidity result and related generalizations and open problems. 


\section{Tits buildings and their geometric realizations}

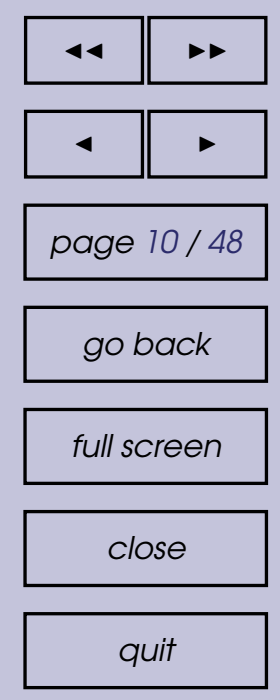

In this section, we recall briefly the notion of spherical buildings and the construction of spherical Tits buildings associated with semisimple real Lie groups. Then we explain a geometric realization of the Tits buildings in terms of the simplicial structures on the boundaries of the geodesic compactifications and their applications to the Mostow strong rigidity of locally symmetric spaces. We also explain how to use Tits buildings to parametrize boundary components of other compactifications of symmetric spaces. Then we recall the Tits building of a semisimple algebraic group G defined over $\mathbb{Q}$ and its relationship with the Tits building of the Lie group $G=\mathbf{G}(\mathbb{R})$.

In the study of a semisimple real Lie group $G$, an important role is played by a finite group, the Weyl group $W$. Briefly, let $G=N A K$ be the Iwasawa decomposition of $G$, let $\mathcal{N}_{K}(A)$ be the normalizer of $A$ in $K$ and let $\mathcal{Z}_{K}(A)$ be the centralizer of $A$ in $K$. Then the quotient $\mathcal{N}_{K}(A) / \mathcal{Z}_{K}(A)$ is the Weyl group $W$ of $G$. It is known that $W$ is generated by elements of order 2 , which correspond to reflections in flats in $X$ with respect to the so-called root hyperplanes.

Let $\mathfrak{a}$ be the Lie algebra of $A$. Then $\mathcal{N}_{K}(A)$ and $\mathcal{Z}_{K}(A)$ can be canonically identified with the normalizer and centralizer of $\mathfrak{a}$ in $K$ respectively.

The Killing form of $\mathfrak{g}$ restricts to an inner product on $\mathfrak{a}$, and $W$ acts isometrically on $\mathfrak{a}$. The roots of the Lie algebra $\mathfrak{g}$ of $G$ with respect to $\mathfrak{a}$ are linear functionals on $\mathfrak{a}$. Each root defines a root hyperplane, and the collection of these hyperplanes is invariant under $W$.

It is known that the reflections with respect to these root hyperplanes generate the Weyl group $W$. The Weyl group is an important example of a finite Coxeter group.

There is a finite complex naturally associated to the action of $W$ on a. In fact, the root hyperplanes and their intersections give a decomposition of $\mathfrak{a}$ into simplicial cones, i.e., the Weyl chambers and Weyl chamber faces. This decomposition is called the Weyl chamber decomposition of $\mathfrak{a}$. The intersection of these simplicial cones with the unit sphere of $\mathfrak{a}$ gives a finite simplicial complex, which is called the Coxeter complex associated with the Weyl group and its underlying topological space is the unit sphere.

In general, for every finite group Coxeter group, there is associated a finite simplicial complex, called the Coxeter complex of the Coxeter group.

With these preparations, we are ready to define spherical Tits buildings.

Definition 3.1. A simplicial complex $\Delta$ is called a spherical Tits building if it contains a family of sub-complexes called apartments, which satisfies the following conditions: 


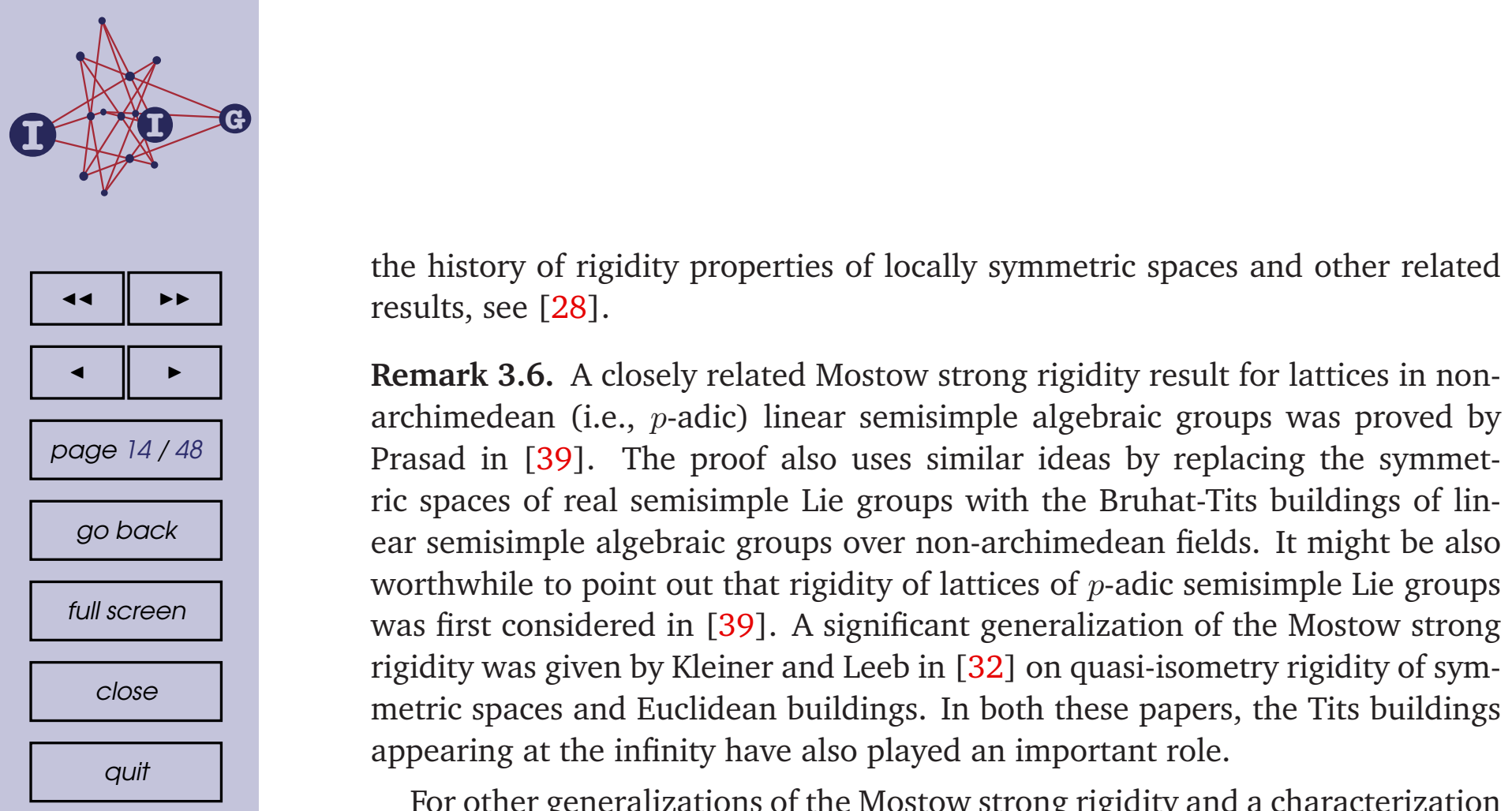
results, see [28].

Remark 3.6. A closely related Mostow strong rigidity result for lattices in nonarchimedean (i.e., $p$-adic) linear semisimple algebraic groups was proved by Prasad in [39]. The proof also uses similar ideas by replacing the symmetric spaces of real semisimple Lie groups with the Bruhat-Tits buildings of linear semisimple algebraic groups over non-archimedean fields. It might be also worthwhile to point out that rigidity of lattices of $p$-adic semisimple Lie groups was first considered in [39]. A significant generalization of the Mostow strong rigidity was given by Kleiner and Leeb in [32] on quasi-isometry rigidity of symmetric spaces and Euclidean buildings. In both these papers, the Tits buildings appearing at the infinity have also played an important role.

For other generalizations of the Mostow strong rigidity and a characterization of irreducible symmetric spaces and Euclidean buildings of higher rank by their asymptotic (or Tits) geometry, see the paper of Leeb [34].

Relationships similar to that in Proposition 3.2 between Tits buildings (or equivalently parabolic subgroups) and boundaries of compactifications can also be established for other compactifications of symmetric spaces. The importance of such relationships is that parabolic subgroups can be used to understand the structure at infinity of $X$. For a systematical discussion, see the books [10, 18].

For a symmetric space $X$ of non-compact type, there are finitely many nonisomorphic Satake compactifications. They are partially ordered, and there is a unique maximal one, called the maximal Satake compactification and denoted by $\bar{X}_{\max }^{S}$.

The maximal Satake compactification $\bar{X}_{\max }^{S}$ is important for many applications. For example, it contains the maximal Furstenberg boundary $G / P_{\min }$, where $P_{\min }$ is a minimal parabolic subgroup of $G$, in its boundary $\partial \bar{X}_{\max }^{S}$, which parametrizes the set of chambers, i.e., simplices of top dimension, of $\Delta(G)$. In the proof of the Mostow strong rigidity theorem outlined above, to show that the map $\varphi_{\infty}$ induces an isomorphism between $\Delta(G)$ and $\Delta\left(G^{\prime}\right)$, the starting pointing is to prove that $\varphi_{\infty}$ maps the maximal Furstenberg boundary of $X$ to the maximal Furstenberg boundary of $X^{\prime}$.

The compactification $\bar{X}_{\max }^{S}$ is also important in determining the Martin compactification of $X$, which deals with structures of the cone of positive eigenfunctions on $X$, for example, how to represent a general positive eigenfunction as a superposition of extremal ones of the cone (see [18]).

The boundary of $\bar{X}_{\max }^{S}$ can be described as follows. With respect to a fixed maximal compact subgroup $K$, every proper parabolic subgroup $P$ of $G$ admits 



\section{$1 \frac{1}{1}=0$
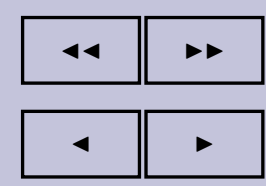 \\ page $16 / 48$ \\ go back \\ full screen \\ close \\ spherical Tits building $\Delta_{\mathbb{Q}}(\mathbf{G})$ associated with $\mathbf{G}$ which also encodes the fact that $\mathbf{G}$ is defined over $\mathbb{Q}$. \\ Assume that $\mathbf{G}$ contains at least one proper parabolic subgroup defined over $\mathbb{Q}$, which is equivalent to assuming that the $\mathbb{Q}$-rank of $\mathbf{G}$ is positive, which is in turn equivalent to assuming that quotients of $X$ by arithmetic subgroups of $\mathbf{G}(\mathbb{Q})$ are noncompact (see Proposition 4.4 below). Otherwise, the building $\Delta_{\mathbb{Q}}(\mathbf{G})$ is empty. Under this assumption, the definition of $\Delta_{\mathbb{Q}}(\mathbf{G})$ is similar to that of $\Delta(G)$ by considering only parabolic subgroups of $\mathbf{G}$ defined over $\mathbb{Q}$, which are often called $\mathbb{Q}$-parabolic subgroups. \\ Specifically, $\Delta_{\mathbb{Q}}(\mathbf{G})$ is an infinite simplicial complex satisfying the following properties:}

quit

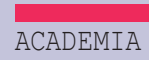

PRESS

WW

$\widehat{\underline{\text { IIIIII }}}$

UNIVERSITEIT

GENT
(1) The simplices of $\Delta_{\mathbb{Q}}(\mathbf{G})$ are parametrized by the set of all proper $\mathbb{Q}$-parabolic subgroups of $\mathbf{G}$. For each $\mathbb{Q}$-parabolic subgroup $\mathbf{P}$, denote the corresponding simplex by $\sigma_{\mathbf{P}}$.

(2) The vertices (i.e. simplices of dimension 0) of $\Delta_{\mathbb{Q}}(\mathbf{G})$ are parametrized by the maximal proper $\mathbb{Q}$-parabolic subgroups of $\mathbf{G}$.

(3) Let $\mathbf{P}_{1}, \ldots, \mathbf{P}_{k}$ be distinct maximal proper parabolic subgroups of $\mathbf{G}$. Then their corresponding zero dimensional simplices form the vertices of a $(k-$ 1)-simplex $\sigma_{\mathbf{P}}$ if and only if the intersection $\mathbf{P}_{1} \cap \cdots \cap \mathbf{P}_{k}$ is equal to the parabolic subgroup $\mathbf{P}$.

In addition, a natural collection of apartments in $\Delta_{\mathbb{Q}}(\mathbf{G})$ can be described in a manner similar to the description of the apartments of $\Delta(G)$. Briefly, for every maximal $\mathbb{Q}$-split torus $\mathbf{T}$ of $\mathbf{G}$, there are only finitely many $\mathbb{Q}$-parabolic subgroups $\mathbf{P}_{1}, \ldots, \mathbf{P}_{m}$ containing $\mathbf{T}$, and their simplices $\sigma_{\mathbf{P}_{1}}, \ldots, \sigma_{\mathbf{P}_{m}}$ form an apartment of $\Delta_{\mathbb{Q}}(\mathbf{G})$.

Remark 3.7. It might be helpful to point out some differences between the two buildings $\Delta_{\mathbb{Q}}(\mathbf{G})$ and $\Delta(G)$ associated with $\mathbf{G}$. It is true that for every proper $\mathbb{Q}$-parabolic subgroup $\mathbf{P}$ of $\mathbf{G}$, its real locus $P=\mathbf{P}(\mathbb{R})$ is a proper parabolic subgroup of $G$, and hence there a simplex $\sigma_{P}$ in $\Delta(G)$ corresponding to $\mathbf{P}$. On the other hand, by the definition of $\Delta_{\mathbb{Q}}(\mathbf{G})$, there is also a simplex $\sigma_{\mathbf{P}}$ of $\Delta_{\mathbb{Q}}(\mathbf{G})$ associated with $\mathbf{P}$. In general, these two simplices $\sigma_{P}$ and $\sigma_{\mathbf{P}}$ are not equal to each other. In particular, $\operatorname{dim} \sigma_{P} \geq \operatorname{dim} \sigma_{\mathbf{P}}$, and the equality holds if and only if the $\mathbb{Q}$-rank of $\mathbf{P}$ is equal to its $\mathbb{R}$-rank, which holds when $\mathbf{P}$ is $\mathbb{Q}$-split. Therefore, if $\mathbf{G}$ is $\mathbb{Q}$-split, then $\Delta_{\mathbb{Q}}(\mathbf{G})$ can be naturally contained in $\Delta(G)$ as a sub-building. Otherwise, there is in general no inclusion between them.

Though these two buildings $\Delta(G)$ and $\Delta_{\mathbb{Q}}(\mathbf{G})$ are different, they have the following property, the so-called Solomon-Tits Theorem (see [14]). 


\section{$1 \frac{1}{1} 0$
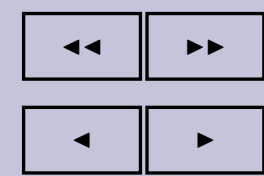 \\ page $17 / 48$ \\ go back \\ full screen \\ Proposition 3.8. Let $r$ be the rank of a semisimple Lie group $G$. Then the Tits building $\Delta(G)$ is homotopic to a bouquet of uncountably many spheres of dimen- sion $r-1$. Let $r_{\mathbb{Q}}$ be the $\mathbb{Q}$-rank of a semisimple linear algebraic group $\mathbf{G}$ defined over $\mathbb{Q}$. Then the Tits building $\Delta_{\mathbb{Q}}(\mathbf{G})$ is homotopic to a bouquet of infinitely but countably many spheres of dimension $r_{\mathbb{Q}}-1$. \\ 4. Arithmetic groups, Borel-Serre compactifications, and Tits buildings}

close

quit

ACADEMIA

PRESS

W

$\widehat{\text { IIIIII }}$

UNIVERSITEIT

GENT
In this section we first recall the definitions of arithmetic subgroups $\Gamma$ of linear algebraic groups $\mathbf{G}$ and of classifying (or universal) spaces for $\Gamma$. Then we recall the Borel-Serre partial compactification $\bar{X}^{\mathrm{BS}}$ of a symmetric space $X$ and an application of this compactification to understanding the ends of locally symmetric spaces $\Gamma \backslash X$. Finally we summarize duality properties of arithmetic subgroups and explain that $\bar{X}^{\mathrm{BS}}$ is a $\Gamma$-cofinite universal space for proper actions of $\Gamma$.

Let $\mathbf{G} \subset \mathrm{GL}(n, \mathbb{C})$ be a semisimple linear algebraic group defined over $\mathbb{Q}$. Then a subgroup $\Gamma \subset \mathbf{G}(\mathbb{Q})$ is called an arithmetic subgroup if it is commensurable with $\mathbf{G}(\mathbb{Z})=\mathbf{G}(\mathbb{Q}) \cap \mathrm{GL}(n, \mathbb{Z})$, i.e., the intersection $\Gamma \cap \mathbf{G}(\mathbb{Z})$ is of finite index in both $\Gamma$ and $\mathbf{G}(\mathbb{Z})$.

As above, let $G=\mathbf{G}(\mathbb{R})$, and let $K \subset G$ be a maximal compact subgroup. Then $X=G / K$ with an invariant metric is a Riemannian symmetric space of non-compact type.

It is easy to see that an arithmetic subgroup $\Gamma$ is a discrete subgroup of $G$ and hence acts properly on $X$. If $\Gamma$ is torsion-free, then the action of $\Gamma$ on $X$ is proper and fixed point free, and $\Gamma \backslash X$ is a manifold.

Proposition 4.1. If $\Gamma$ is a torsion-free arithmetic subgroup, then the locally symmetric space $\Gamma \backslash X$ is a $K(\Gamma, 1)$-space.

Proof. By definition, a $K(\Gamma, 1)$-space is a topological space $B$ with $\pi_{1}(B)=\Gamma$ and $\pi_{i}(B)=\{1\}$ for $i \geq 2$. Since $X$ is contractible and the action of $\Gamma$ is proper and fixed point free, $\pi_{1}(\Gamma \backslash X)=\Gamma$ and for $i \geq 2, \pi_{i}(\Gamma \backslash X)=\pi_{i}(X)=\{1\}$.

The existence of a good model of $K(\Gamma, 1)$ has important consequences on cohomological properties of $\Gamma$. An immediately corollary is the following result.

Corollary 4.2. If $\Gamma$ is a torsion-free arithmetic subgroup, then the cohomological dimension of $\Gamma$ is less than or equal to $\operatorname{dim} X$, i.e., for every $\Gamma$-module $M$, $H^{i}(\Gamma, M)=0$ for $i>\operatorname{dim} X$. 
To get other finiteness properties such as $F P_{\infty}$ or $F L$ (see [14]), we need some finiteness conditions on models of $K(\Gamma, 1)$. A strong finiteness condition is that there exists a finite $K(\Gamma, 1)$-space in the following sense.

Definition 4.3. A $K(\Gamma, 1)$-space is called finite if it is given by a finite CW-complex.

If $\Gamma$ is torsion-free and the quotient $\Gamma \backslash X$ is compact, then $\Gamma \backslash X$ is a compact manifold and hence admits a finite triangulation. This implies that $\Gamma$ admits a finite $K(\Gamma, 1)$-space.

Therefore, a natural and important problem is to determine when the quotient $\Gamma \backslash X$ is compact, i.e., when $\Gamma$ is a uniform lattice subgroup of $G$.

Proposition 4.4. Let $\mathbf{G}$ be a semisimple linear algebraic group defined over $\mathbb{Q}$. Then for every arithmetic subgroup $\Gamma \subset \mathbf{G}(\mathbb{Q}), \Gamma \backslash X$ is compact if and only if the $\mathbb{Q}$-rank of $\mathbf{G}$ is equal to zero, or equivalently there is no proper $\mathbb{Q}$-parabolic subgroup of $\mathbf{G}$.

This was conjectured by Godement and proved by Borel and Harish-Chandra [9], and by Mostow and Tamagawa [37].

If $\Gamma \backslash X$ is non-compact, then $\mathbf{G}$ admits proper $\mathbb{Q}$-parabolic subgroups, or equivalently the Tits building $\Delta_{\mathbb{Q}}(\mathbf{G})$ is nontrivial, which turns out to be crucial in describing the boundary at infinity of $\Gamma \backslash X$.

When $\Gamma \backslash X$ is non-compact, it certainly is not a finite CW-complex. One natural way to overcome this problem is to find a compactification $\overline{\Gamma \backslash X}$ such that

(1) the compactification $\overline{\Gamma \backslash X}$ has the structure of a finite CW-complex,

(2) and the inclusion $\Gamma \backslash X \rightarrow \overline{\Gamma \backslash X}$ is a homotopy equivalence.

The first condition is satisfied if $\overline{\Gamma \backslash X}$ is a compact manifold with boundary or with corners, and with interior equal to $\Gamma \backslash X$.

When $X$ is the Poincaré upper halfplane $\mathbb{H}^{2}$, a non-compact quotient $\Gamma \backslash X$ is a Riemann surface with finitely many cusp neighborhoods. Each cusp neighborhood is diffeomorphic to a cylinder $[0,+\infty) \times S^{1}$ and hence can be compactified by adding a circle $S^{1}$ at infinity. The resulting compactification $\overline{\Gamma \backslash X}$ of $\Gamma \backslash X$ is a compact surface with boundary, and the inclusion $\Gamma \backslash X \rightarrow \overline{\Gamma \backslash X}$ is clearly a homotopy equivalence.

For a general non-compact locally symmetric space $\Gamma \backslash X$ associated to an arithmetic subgroup $\Gamma$, Borel and Serre [11] defined a compactification $\overline{\Gamma \backslash X}^{\mathrm{BS}}$ of $\Gamma \backslash X$ which has similar properties as follows. 

(2) If $r_{\mathbb{Q}}>1$, then $\Gamma \backslash X$ has only one end, i.e., the infinity of $\Gamma \backslash X$ is connected.

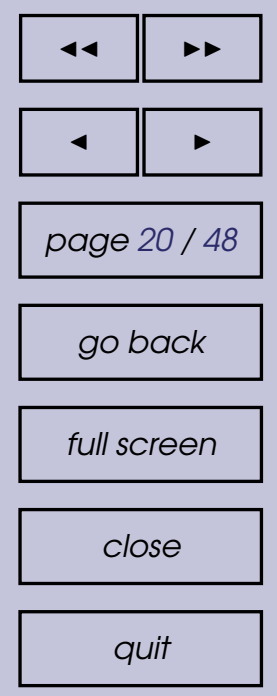

Proof. By definition, the ends of a complete connected noncompact Riemannian manifold $M$ are basically the unbounded connected components of the complement of compact subsets. Specifically, for any compact subset $C \subset M$, denote by $n(C)$ the number of unbounded connected components of the complement $M-C$. Clearly $n(C)<+\infty$. For any two compact subsets $C_{1}, C_{2}$ of $M$ with $C_{1} \subset C_{2}$, it is also clear that $n\left(C_{1}\right) \leq n\left(C_{2}\right)$. The number $e(M)$ of ends of $M$ is defined by

$$
e(M)=\sup _{C} n(C)
$$

where $C$ ranges over compact subsets of $M$.

Assume that $e(M)<+\infty$. Then there exists a compact subset $C_{0}$ such that $n\left(C_{0}\right)=e(M)$, and the unbounded connected components of $M-C_{0}$ are called the ends of $M$. Clearly we can replace $C_{0}$ by any larger compact subset and hence ends of $M$ are well-defined up to compact subsets.

First we assume that $r_{\mathbb{Q}} \geq 2$ and that $\Gamma$ is torsion-free. In this case, the Tits building $\Delta_{\mathbb{Q}}(\mathbf{G})$ is connected. It suffices to show that for every compact subset $C$ of $\Gamma \backslash X$, the complement $\Gamma \backslash X-C$ has only one unbounded connected component. Since $\overline{\Gamma \backslash X}{ }^{\mathrm{BS}}$ is a manifold with corners with the interior equal to $\Gamma \backslash X$, for every compact subset $C$ of $\Gamma \backslash X$, there is a neighborhood $U$ of the boundary $\partial \overline{\Gamma \backslash X}^{\mathrm{BS}}$ such that the complement $\overline{\Gamma \backslash X}^{\mathrm{BS}}-U$ contains $C$ and $U$ is homotopic to the boundary $\partial \overline{\Gamma \backslash X}^{\mathrm{BS}}$ by deformation retraction. Since $\partial \bar{X}^{\mathrm{BS}}$ has the same homotopy type as the building $\Delta_{\mathbb{Q}}(\mathbf{G})$, and $\Delta_{\mathbb{Q}}(\mathbf{G})$ is connected, it follows that $\partial \overline{\Gamma \backslash X}^{\mathrm{BS}}=\Gamma \backslash \partial \bar{X}^{\mathrm{BS}}$ and hence $U$ is connected. Since $U \cap \Gamma \backslash X$ and $U$ are also homotopic to each other, $U \cap \Gamma \backslash X$ is also connected. Note that the complement $\Gamma \backslash X-C$ of $C$ in $\Gamma \backslash X$ is contained in $U \cap \Gamma \backslash X$, and the complement of $U \cap \Gamma \backslash X$ in $\Gamma \backslash X$ is compact. It follows that the complement $\Gamma \backslash X-C$ has only one unbounded connected component. By the arbitrary choice of $C$, this implies that $\Gamma \backslash X$ has only one end.

If $\Gamma$ contains some nontrivial torsion elements, then there is always a torsionfree normal subgroup $\Gamma^{\prime}$ of finite index. By the previous paragraph, the locally symmetric space $\Gamma^{\prime} \backslash X$ has only one end. Since $\Gamma^{\prime} \backslash X$ is a finite cover of $\Gamma \backslash X$, any compact subset of $\Gamma \backslash X$ is lifted to a compact subset of $\Gamma^{\prime} \backslash X$. This implies that $\Gamma \backslash X$ has also only one end.

Before treating the case when the rank $r_{\mathbb{Q}}=1$, we recall the rational Langlands decomposition and Siegel sets. For every $\mathbb{Q}$-parabolic subgroup $\mathbf{P}$ of $\mathbf{G}$, there is also a rational Laglands decomposition of the real locus $P=\mathbf{P}(\mathbb{R})$ with respect to the maximal compact subgroup $K \subset G$ obtained by taking the 







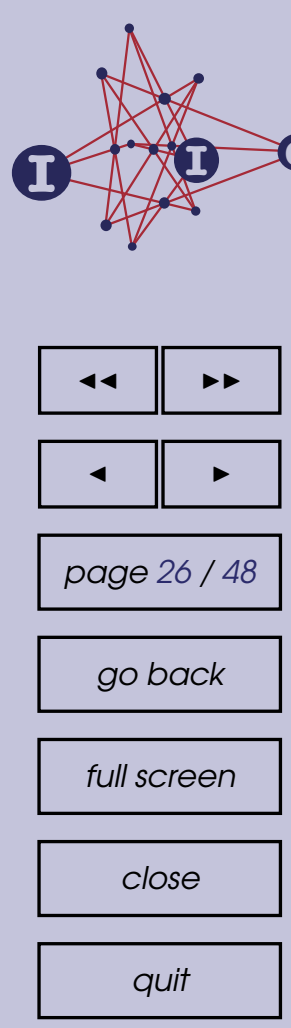

simplicial complex whose simplices are parametrized by parabolic subgroups of G defined over $\mathbb{Q}_{p}$ and satisfy properties similar to those for the Tits building $\Delta(G)$ of a semisimple real Lie group $G$ in $\S 3$. Since $\mathbf{G}\left(\mathbb{Q}_{p}\right)$ acts on the set of $\mathbb{Q}_{p}$-parabolic subgroups by conjugation, it also acts on the building $\Delta\left(\mathbf{G}\left(\mathbb{Q}_{p}\right)\right)$.

The analogue of the symmetric space $X=G / K$ for $\mathbf{G}\left(\mathbb{Q}_{p}\right)$ is the Bruhat-Tits building, denoted by $\Delta^{\mathrm{BT}}\left(\mathbf{G}\left(\mathbb{Q}_{p}\right)\right)$ in this paper, which is a Euclidean building (also called an affine building).

The definition and construction of $\Delta^{\mathrm{BT}}\left(\mathbf{G}\left(\mathbb{Q}_{p}\right)\right)$ is much more complicated than that of the spherical Tits building $\Delta\left(\mathbf{G}\left(\mathbb{Q}_{p}\right)\right.$. To motivate the Bruhat-Tits building $\Delta^{\mathrm{BT}}\left(\mathbf{G}\left(\mathbb{Q}_{p}\right)\right)$, we note that for the semisimple Lie group $G=\mathbf{G}(\mathbb{R})$, its symmetric space $X=G / K$ can be identified with the space of maximal compact subgroups of $G$. An important fact here is that all maximal compact subgroups of $G$ are conjugate. But this statement is not true for $\mathbf{G}\left(\mathbb{Q}_{p}\right)$. In general, there is more than one conjugacy class of maximal compact subgroups of $\mathbf{G}\left(\mathbb{Q}_{p}\right)$.

For simplicity, we assume that $\mathbf{G}$ is a simply connected semisimple linear algebraic group defined over $\mathbb{Q}$. Let $r$ be the $\mathbb{Q}_{p}$-rank of $\mathbf{G}$. In this case, there are exactly $(r+1)$-conjugacy classes of maximal compact open subgroups of $\mathbf{G}\left(\mathbb{Q}_{p}\right)$. Let $P_{1}, \ldots, P_{r+1}$ be representatives of such conjugacy classes. Then it is natural to consider the homogeneous spaces $\mathbf{G}\left(\mathbb{Q}_{p}\right) / P_{1}, \ldots, \mathbf{G}\left(\mathbb{Q}_{p}\right) / P_{r+1}$, each of which is a disjoint union of points. For various purposes, it is desirable to enhance them into simplicial complexes so that they remain as vertices and then to combine these finitely many simplicial complexes suitably into one single simplicial complex. Basically, the Bruhat-Tits building $\Delta^{\mathrm{BT}}\left(\mathbf{G}\left(\mathbb{Q}_{p}\right)\right)$ can be visualized this way.

One original way to construct the Bruhat-Tits building $\Delta^{\mathrm{BT}}\left(\mathbf{G}\left(\mathbb{Q}_{p}\right)\right)$ is to use the method of BN-pairs and, instead of using parabolic subgroups of $G(\mathbb{R})$ as was done for the spherical Tits building $\Delta(G)$, to use the so-called parahoric groups. This depends crucially on the fact that $\mathbb{Q}_{p}$ has a nontrivial discrete valuation.

Under the assumption that $\mathbf{G}$ is simply connected, maximal compact open subgroups of $\mathbf{G}\left(\mathbb{Q}_{p}\right)$ are maximal parahoric subgroups, and maximal parahoric subgroups are also maximal compact open subgroups of $\mathbf{G}\left(\mathbb{Q}_{p}\right)$. More importantly, the structure of parahoric subgroups $\mathbf{G}\left(\mathbb{Q}_{p}\right)$ is similar to the structure of parabolic subgroups of $\mathbf{G}(\mathbb{R})$. For example, the following result reminds one of the structure of parabolic subgroups of $G$ and the notion of standard parabolic subgroups (see [25] and references there for more details).

Proposition 5.1. Under the above assumptions on $\mathbf{G}$, all minimal parahoric subgroups of $\mathbf{G}\left(\mathbb{Q}_{p}\right)$ are conjugate. Fix any minimal parahoric subgroup $B$. Then there are exactly $r+1$ maximal parahoric subgroups $P_{1}, \ldots, P_{r+1}$ which contain $B$, and $\left\{P_{i_{1}} \cap \cdots \cap P_{i_{j}}\right\}$ are exactly the parahoric subgroups which contain $B$ when 


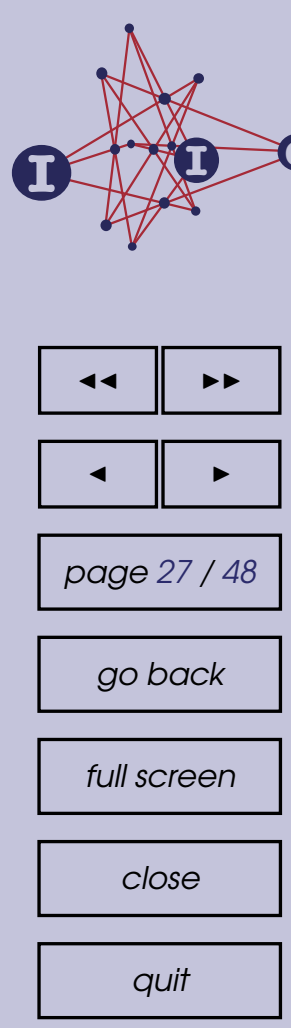

$\left\{i_{1}, \ldots, i_{j}\right\}$ runs through non-empty subsets of $\{1, \ldots, r+1\}$. They are called the standard parahoric subgroups. Furthermore, any parahoric subgroup of $\mathbf{G}\left(\mathbb{Q}_{p}\right)$ is conjugate to such a standard parahoric subgroup.

Bruhat-Tits buildings are important examples of Euclidean buildings. We recall some of their basic properties. See [15] for more details.

Recall from $\S 3$ that an important notion in the definition of a spherical Tits buildings is that of apartments, which are finite Coxeter complexes. Recall that their underlying topological spaces are spheres, and that buildings are obtained by gluing these apartments together suitably. For Euclidean buildings, finite Coxeter complexes are replaced by infinite Euclidean Coxeter complexes, whose underlying spaces are Euclidean spaces.

Briefly, let $V$ be a Euclidean space. An affine reflection group $W$ on $V$ is a group of affine isometries generated by reflections with respect to affine hyperplanes such that the set $\mathcal{H}$ of affine hyperplanes fixed by reflections in $W$ is locally finite.

The linear parts of the affine transformations in $W$ define a finite (linear) reflection group $\bar{W}$, or a finite Coxeter group. An infinite affine reflection group is called a Euclidean reflection group.

The hyperplanes in $\mathcal{H}$ divide $V$ into chambers, and $W$ acts simply transitively on the set of chambers. If the reflection group $W$ is irreducible, then the chambers and their faces form a simplicial complex. Otherwise, they form a polysimplicial complex which is a product of simplicial complexes. Such a polysimplicial complex is called a Euclidean Coxeter complex. For simplicity, in the following we assume that the affine reflection groups $W$ and the Euclidean Bruhat-Tits buildings introduced below are irreducible. Otherwise, we get polysimplicial complexes instead of simplicial complexes.

Definition 5.2. A polysimplicial complex $\Delta$ is called a Euclidean building if it contains a family of subsets called apartments, which satisfies the following conditions:

(1) Every apartment is an infinite Euclidean Coxeter complex.

(2) Any two simplices are contained in some apartment.

(3) Given two apartments $\Sigma$ and $\Sigma^{\prime}$ and simplices $\sigma, \sigma^{\prime} \in \Sigma \cap \Sigma^{\prime}$, there exists an isomorphism of $\Sigma$ onto $\Sigma^{\prime}$ which keeps $\sigma, \sigma^{\prime}$ pointwise fixed.

The Bruhat-Tits building $\Delta^{\mathrm{BT}}\left(\mathbf{G}\left(\mathbb{Q}_{p}\right)\right)$ is a Euclidean building, and its simplices are parametrized by parahoric subgroups of $\mathbf{G}\left(\mathbb{Q}_{p}\right)$ such that maximal parahoric subgroups correspond to vertices, i.e., simplices of zero dimension, 


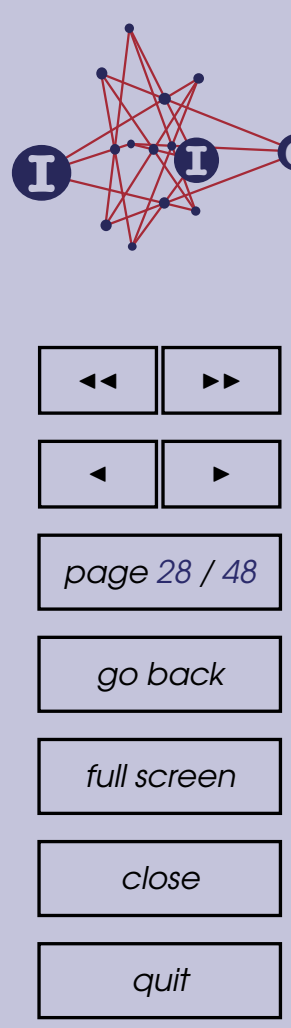

and minimal parahoric subgroups correspond to chambers, i.e., top dimensional simplices. Since $\mathbf{G}\left(\mathbb{Q}_{p}\right)$ acts on the set of parahoric subgroups by conjugation, it acts on $\Delta^{\mathrm{BT}}\left(\mathbf{G}\left(\mathbb{Q}_{p}\right)\right)$.

Since the underlying space of each Euclidean Coxeter complex is a Euclidean space, it has a metric, or a distance function. Fix a Euclidean metric on every apartment such that all apartments are isometric and their induced metrics agree on their intersections. Then these metrics can be glued to give a metric on the building $\Delta^{\mathrm{BT}}\left(\mathbf{G}\left(\mathbb{Q}_{p}\right)\right)$ which becomes a geodesic space (recall that a geodesic space is a metric space such that the distance between any two points is realized by a geodesic connecting them) [14, Chap. VI, §3].

The group $\mathbf{G}\left(\mathbb{Q}_{p}\right)$ acts on the Bruhat-Tits building $\Delta^{\mathrm{BT}}\left(\mathbf{G}\left(\mathbb{Q}_{p}\right)\right)$ by isometries. Furthermore, the action is proper. (Note that the stabilizers of the simplices coincide with their corresponding parahoric subgroups up to finite index, and are hence compact.)

Proposition 5.3. Any Euclidean building $\Delta$ as a metric space is a CAT(0)-space, and hence has nonpositive curvature and is contractible. In particular, it is simply connected.

Recall that a CAT(0)-space $M$ is a geodesic length space such that every triangle in $M$ is thinner than a corresponding triangle in $\mathbb{R}^{2}$ of the same side lengths [13]. This proposition implies that if a compact group acts isometrically on $\Delta$, then it has at least one fixed point, which has important applications to understanding structures of compact open subgroups of $\mathbf{G}\left(\mathbb{Q}_{p}\right)$ as mentioned in Proposition 5.1.

The Bruhat-Tits building $\Delta^{\mathrm{BT}}\left(\mathbf{G}\left(\mathbb{Q}_{p}\right)\right)$ is non-compact. Since it is a proper CAT(0)-space, it admits a compactification by adding the set of equivalence classes of geodesics as was discussed for Hadamard manifolds in $\S 2$. It turns out that this set can be canonically identified with the spherical Tits building $\Delta\left(\mathbf{G}\left(\mathbb{Q}_{p}\right)\right)$. It should be emphasized that the topology on $\Delta\left(\mathbf{G}\left(\mathbb{Q}_{p}\right)\right)$ induced from the compactification is not the simplicial one. See $[12,14]$.

Remark 5.4. As pointed out before, maximal totally geodesic flat subspaces of a symmetric space $X$ play a fundamental role in understanding the geometry of $X$, and $X$ is the union of such flats. Apartments in a Euclidean building, in particular, the Bruhat-Tits building $\Delta^{\mathrm{BT}}\left(\mathbf{G}\left(\mathbb{Q}_{p}\right)\right)$, are also maximal totally geodesic subspaces, and the building is also the union of such flats. Furthermore, both $X$ and $\Delta^{\mathrm{BT}}\left(\mathbf{G}\left(\mathbb{Q}_{p}\right)\right)$ are CAT(0)-spaces and the Cartan fixed point theorem holds for them. In this sense, the Bruhat-Tits building $\Delta^{\mathrm{BT}}\left(\mathbf{G}\left(\mathbb{Q}_{p}\right)\right)$ is a good replacement for the symmetric space $X$. The fact that they can be compactified by adding the spherical buildings of $\mathbf{G}(\mathbb{R})$ and $\mathbf{G}\left(\mathbb{Q}_{p}\right)$ respectively is another indication of their similarity. 


\section{Finiteness properties and duality properties of S-arithmetic groups}

Let $\Gamma \subset \mathbf{G}(\mathbb{Q})$ be an $S$-arithmetic subgroup, i.e., a subgroup commensurable with $\mathbf{G}(\mathbb{Q}) \cap \mathrm{GL}\left(n, \mathbb{Z}\left[\frac{1}{p_{1}}, \ldots, \frac{1}{p_{k}}\right]\right)$ as in the previous section.

By equation (5), $\Gamma$ is a discrete subgroup of $\mathbf{G}(\mathbb{R}) \times \prod_{p \in S} \mathbf{G}\left(\mathbb{Q}_{p}\right)$. Since each $\mathbf{G}\left(\mathbb{Q}_{p}\right)$ acts isometrically and properly on the building $\Delta^{\mathrm{BT}}\left(\mathbf{G}\left(\mathbb{Q}_{p}\right)\right), \Gamma$ acts isometrically and properly on the product

$$
X_{S}=X \times \prod_{p \in S} \Delta^{\mathrm{BT}}\left(\mathbf{G}\left(\mathbb{Q}_{p}\right)\right) .
$$

Since each of the factors is a proper CAT(0)-space, the product $X_{S}$ is also a proper CAT(0)-space. An immediately corollary of this action is the following.

Proposition 5.5. The space $X_{S}$ is an $\underline{E} \Gamma$-space; in particular, if $\Gamma$ is torsion-free, then the quotient $\Gamma \backslash X_{S}$ is a $K(\Gamma, 1)$-space and hence the cohomological dimension of $\Gamma$ is less than or equal to $\operatorname{dim} X_{S}$. If the $\mathbb{Q}$-rank of $\mathbf{G}$ is 0 , then $\Gamma \backslash X_{S}$ is compact, and $X_{S}$ is a $\Gamma$-cofinite $\underline{E} \Gamma$-space.

On the other hand, if the $\mathbb{Q}$-rank of $\mathbf{G}$ is positive, then $\Gamma \backslash X_{S}$ is non-compact. As recalled earlier, $X$ admits the Borel-Serre partial compactification $\bar{X}^{\mathrm{BS}}$. Define a partial compactification of $X_{S}$ by

$$
{\overline{X_{S}}}^{\mathrm{BS}}=\bar{X}^{\mathrm{BS}} \times \prod_{p \in S} \Delta^{\mathrm{BT}}\left(\mathbf{G}\left(\mathbb{Q}_{p}\right)\right) .
$$

Then the $\Gamma$ action on $X_{S}$ extends to ${\overline{X_{S}}}^{\mathrm{BS}}$ and the quotient $\Gamma \backslash{\overline{X_{S}}}^{\mathrm{BS}}$ is compact [12]. The following result of Borel and Serre [12] is an important application of ${\overline{X_{S}}}^{\mathrm{BS}}$ together with the homotopy equivalence between the boundary $\partial \bar{X}^{\mathrm{BS}}$ and the Tits building $\Delta_{\mathbb{Q}}(\mathbf{G})(\S 4)$ and the compactification of the Bruhat-Tits building $\Delta^{\mathrm{BT}}\left(\mathbf{G}\left(\mathbb{Q}_{p}\right)\right)$ by attaching a spherical building $\Delta\left(\mathbf{G}\left(\mathbb{Q}_{p}\right)\right)(\S 5)$.

Theorem 5.6. If $\Gamma$ is a torsion-free $S$-arithmetic subgroup of $\mathbf{G}(\mathbb{Q})$ as above, then $\Gamma$ is a duality group. If the $\mathbb{Q}$-rank of $\mathbf{G}$ is positive, then $\Gamma$ is not a Poincaré duality group.

If $\Gamma$ is torsion-free and the $\mathbb{Q}$-rank of $\mathbf{G}$ is positive, then $\Gamma \backslash{\overline{X_{S}}}^{\mathrm{BS}}$ is a finite $K(\Gamma, 1)$-space. As in the case of arithmetic subgroups, many natural S-arithmetic subgroups contain torsion elements, for example, $\operatorname{SL}\left(n, \mathbb{Z}\left[\frac{1}{p_{1}}, \ldots, \frac{1}{p_{k}}\right]\right)$. A natural problem is to find good models of $\Gamma$-cofinite $E \Gamma$-spaces. By methods similar to the proof of [26, Theorem 3.2] (see Theorem 4.9), we can prove the following.

Proposition 5.7. For a non-uniform S-arithmetic subgroup $\Gamma$ as above, ${\overline{X_{S}}}^{\mathrm{BS}}$ is a $\Gamma$-cofinite $\underline{E} \Gamma$-space. 


\section{Integral Novikov conjectures and the Borel conjecture}

Besides applications to cohomological properties mentioned in the previous section, the action of S-arithmetic subgroups on $X_{S}$ is also important for other results such as the integral Novikov conjecture for S-arithmetic subgroups and the stable Borel conjecture.

In this section, we first recall the Borel conjecture in geometric topology and a weaker version, the stable Borel conjecture. Then we explain both the original version of the Novikov conjecture on homotopy invariance of higher signatures and the integral Novikov conjecture in terms of the modern formulation by the assembly map. Then we explain the integral Novikov conjecture for S-arithmetic subgroups and finitely generated linear groups by making use of their actions on Bruhat-Tits buildings.

An important conjecture in geometric topology is the Borel conjecture, which states that if $M$ and $N$ are two aspherical closed manifolds with the same fundamental group, i.e., they are homotopic, then $M$ and $N$ are homeomorphic.

In the above statement, a manifold $M$ is called aspherical if $\pi_{i}(M)=\{1\}$ for $i \geq 2$. If a $K(\Gamma, 1)$-space can be realized by a closed manifold $M$, then $M$ is an aspherical manifold.

It is an important problem to find conditions under which a $K(\Gamma, 1)$-space can be realized by a closed manifold. The Borel conjecture is basically about the uniqueness up to homeomorphism for such realizations.

Clearly the Borel conjecture only depends on the fundamental group $\pi_{1}(M)$. It is still open and has motivated a lot of work in geometric topology.

A weaker version of the Borel conjecture is the stable Borel conjecture which states that if $M$ and $N$ are two aspherical closed manifolds with the same fundamental group, then $M \times \mathbb{R}^{3}$ and $N \times \mathbb{R}^{3}$ are homeomorphic.

Another closely related conjecture is the integral Novikov conjecture. We briefly recall the motivations and different versions of Novikov conjecture.

The original Novikov conjecture concerns homotopy invariance of higher signatures. Briefly, let $M$ be an oriented closed manifold, and $\Gamma=\pi_{1}(M)$ its fundamental group. Let $B \Gamma$ be the classifying space of $\Gamma$, i.e., a $K(\Gamma, 1)$-space. Let $f: M \rightarrow B \Gamma$ be the classifying map corresponding to the universal covering space $\tilde{M} \rightarrow M$. For any $\alpha \in H^{*}(M, \mathbb{Q})$, define a higher signature

$$
\operatorname{Sgn}_{\alpha}(M)=\left\langle\mathcal{L}(M) \cup f^{*}(\alpha),[M]\right\rangle
$$

associated with $\alpha$, where $\mathcal{L}(M)$ is the Hirzebruch class of $M$. When $\alpha=1$, $\operatorname{Sgn}_{\alpha}(M)$ is equal to the usual signature, by the Hirzebruch index theorem. 





\section{Truncated Teichmüller spaces, Borel-Serre com- pactifications, and curve complexes}

In this section, we define the curve complex $\mathcal{C}(S)$ of a surface $S$ and explain how it is related to the boundary of the Borel-Serre partial compactification ${\overline{T_{g}}}^{\text {BS }}$ of the Teichmüller space $T_{g}$. We give a realization of the Borel-Serre partial compactification $\bar{T}_{g}$ BS by a truncated subspace $T_{g}(\varepsilon)$, and this gives a model of a cofinite universal space for proper actions of $\operatorname{Mod}_{g}$. Then we explain an analogue of the Solomon-Tits theorem for curve complexes and use it together with the truncated subspace $T_{g}(\varepsilon)$ to obtain duality properties of mapping class groups.

It is known that the moduli space $\mathcal{M}_{g}$ is non-compact, and hence the action of $\operatorname{Mod}_{g}^{+}$on $T_{g}$ is similar to the action of a non-umiform arithmetic subgroup $\Gamma$ on the associated symmetric space $X$. This implies that $T_{g}$ is not a $\operatorname{Mod}_{g}^{+}$cofinite $\underline{E} \operatorname{Mod}_{g}^{+}$-space.

In fact, starting with any closed hyperbolic metric, we can pinch along a simple closed geodesic and produce a family of hyperbolic surfaces in $\mathcal{M}_{g}$ which does not have any accumulation point in $\mathcal{M}_{g}$. More specifically, fix any simple closed curve $c$ in $S$. For each marked hyperbolic surface $(\Sigma, d s ; \varphi), \varphi(c)$ gives a homotopy class of simple closed curves in $\Sigma$. Since $(\Sigma, d s)$ has strictly negative sectional curvature, there is a unique closed geodesic in this homotopy class. Denote the length of this geodesic by $\ell_{\Sigma}(c)$.

Then for any $\varepsilon$ sufficiently small, the marked hyperbolic $(\Sigma, d s ; \varphi)$ can be deformed to a marked hyperbolic metric $\left(\Sigma_{\varepsilon}, d s_{\varepsilon} ; \varphi\right)$ such that $\ell_{\Sigma_{\varepsilon}}(c)=\varepsilon$. Certainly, the image in $\mathcal{M}_{g}$ of this family of hyperbolic surfaces $\left(\Sigma_{\varepsilon}, d s_{\varepsilon} ; \varphi\right)$ has no accumulation point in $M_{g}$.

Similarly, we can also pinch along several disjoint simple closed geodesics. It turns out that pinching along geodesics is the only reason for the noncompactness of the quotient $\operatorname{Mod}_{g}^{+} \backslash T_{g}$. Specifically, for any small fixed positive constant $\varepsilon$, define a subspace $T_{g}(\varepsilon)$ by

$$
T_{g}(\varepsilon)=\left\{(\Sigma, d s ; \varphi) \mid \ell_{\Sigma}(c) \geq \varepsilon, \text { for every simple closed curve } c\right\} .
$$

Clearly $T_{g}(\varepsilon)$ is invariant under $\operatorname{Mod}_{g}$. It is known that the quotient $\operatorname{Mod}_{g} \backslash T_{g}(\varepsilon)$ is compact [38].

Motivated by symmetric and locally symmetric spaces, a natural question is that of how to compactify $T_{g}$ and its quotients for various applications, for example, to get an analogue of the Borel-Serre compactification, which will give a cofinite $\underline{E} \operatorname{Mod}_{g}$-space. 
In fact, an analogue of the Borel-Serre compactification was constructed by Harvey [21]. He outlined a construction of a partial compactification of $T_{g}$ which is a real analytic manifold with corners, denoted by ${\overline{T_{g}}}^{\text {BS }}$, such that the quotient $\operatorname{Mod}_{g} \backslash{\overline{T_{g}}}^{\mathrm{BS}}$ is compact.

The boundary $\partial{\overline{T_{g}}}^{\text {BS }}$ consists of contractible pieces that are parametrized by an infinite simplicial complex, called the curve complex of $S$, and denoted by $\mathcal{C}(S)$.

Briefly, for each simple closed curve $c$ in $S$, denote by $[c]$ the homotopy class of $c$. Then the vertices of $\mathcal{C}(S)$ correspond to homotopy classes $[c]$ of simple closed curves. Any collection of distinct homtopy classes $\left[c_{1}\right], \ldots,\left[c_{k}\right]$, forms the vertices of a $(k-1)$-simplex if and only if they contain disjoint representatives.

Since we can pinch along disjoint simple closed geodesics to go to the boundary at infinity of $\operatorname{Mod}_{g} \backslash T_{g}$ and this is basically the only way to go to infinity, it is reasonable that the curve complex $\mathcal{C}(S)$ describes the structure at infinity of $T_{g}$ and its quotients such as $M_{g}$. For example, the curve complex was used in the original application of [21] to parametrize the boundary components of ${\overline{T_{g}}}^{\mathrm{BS}}$. In this sense, it is an analogue of the spherical Tits buildings for symmetric spaces.

As in the case of the Borel-Serre compactification $\bar{X}^{\mathrm{BS}}$ for symmetric spaces, $\bar{T}_{g}$ BS can also be realized by the truncated subspace $T_{g}(\varepsilon)$. See [23] for details and references. In fact, it is easy to see that $T_{g}(\varepsilon)$ is a manifold with corners, and its boundary faces are parametrized by simplices of $\mathcal{C}(S)$.

The following result can also be proved [31].

Theorem 8.1. The truncated space $T_{g}(\varepsilon)$ is a $\operatorname{Mod}_{g}$-cofinite $\underline{E} \operatorname{Mod}_{g}$-space.

As mentioned earlier, in some applications, the Solomon-Tits theorem on the homotopy type of spherical Tits buildings is important. An analogue of this is also true.

Theorem 8.2. The curve complex $\mathcal{C}(S)$ has the homotopy type of bouquet spheres of dimension $2 g-2$. Furthermore, there are infinitely many spheres in the bouquet.

The first statement was proved by Harvey (see [20]), and the statement that the bouquet contains at least one sphere and the statement that its contains infinitely many spheres were proved recently in [24].

By combining the above theorem and the relation between ${\overline{T_{g}}}^{\text {BS }}$ (or rather $T_{g}(\varepsilon)$ ) and $\mathcal{C}(S)$, the following result can be proved (see [20, 23, 24]).

Theorem 8.3. Any torsion free, finite index subgroup of the mapping class group $\operatorname{Mod}_{g}$ is a duality group, but is not a Poincaré duality group. 



In the following, we outline the construction of a truncated subspace $X_{n}(\varepsilon)$ of $X$, which is similar to the truncated symmetric space $X_{T}$ and the truncated Teichmüller space $T_{g}(\varepsilon)$.

It was pointed out before that these truncated spaces are realizations of the Borel-Serre partial compactifications of $X$ and $T_{g}$ respectively. It can be shown that the space $X_{n}(\varepsilon)$ is also $\operatorname{Out}\left(F_{n}\right)$-equivariantly homeomorphic by cellular maps to the Borel-Serre partial compactification $\bar{X}_{n}$ BS constructed in [8].

We also outline the construction of a simplicial complex, called the core graph complex, $\mathcal{C G}\left(F_{n}\right)$, which is isomorphic to the complex of free factors of [22], and show how this core graph complex can be used to parametrize boundary components of $X_{n}(\varepsilon)$. Therefore, this provides an analogue to the relationship between the spherical Tits building $\Delta_{\mathbb{Q}}(\mathbf{G})$ and the boundary of the Borel-Serre partial compactification $\bar{X}^{\mathrm{BS}}$.

In the definition of $T_{g}(\varepsilon)$, simple closed geodesics play an important role. The key point in defining a truncated outer space $X_{n}$ is to find a replacement for collections of disjoint simple closed geodesics in surfaces for metric graphs.

A natural analogue of a simple closed geodesic in a graph $G$ is a subgraph of $G$ that contains no nodes of valence 0 or 1 , which is roughly speaking a loop without spikes coming out.

But there is an important difference between closed geodesics in hyperbolic surfaces and the above loops in graphs: If two simple closed geodesics in a surface agree on any small segment, then they agree everywhere; but this is not true for the loops in graphs.

In the boundary of $T_{g}(\varepsilon)$, boundary faces of codimension 1 are defined by requiring that exactly one marked simple closed geodesic has length equal to $\varepsilon$, and boundary faces of higher codimension $k \geq 2$ are defined by requiring that exactly $k$ marked disjoint simple closed geodesics have length equal to $\varepsilon$.

To obtain an analogue to the conditions for the boundary face of $T_{g}(\varepsilon)$ induced from the lengths of more than one more simple closed geodesics to get boundary faces of higher codimension, we need to use the notion of core subgraphs. Following [8], a core subgraph $C$ of a graph $G$ is a subgraph which contains no nodes of valence 0 or 1 , or separating edges. It is not necessarily connected. Roughly, $C$ is the union of subgraphs without isolated nodes or spikes, or more intuitively a union of loops which could have overlaps on some edges. An important invariant of a core subgraph $C$ is the rank, and is denoted by $r(C)$ : if the connected components of $C$ are joined by minimal number of bridges to get a new connected subgraph $\tilde{C}$, then $r(C)$ is defined to be the rank of the fundamental group $\pi_{1}(\tilde{C})$. Roughly speaking, the rank $r(C)$ is equal to the number of loops in it. 





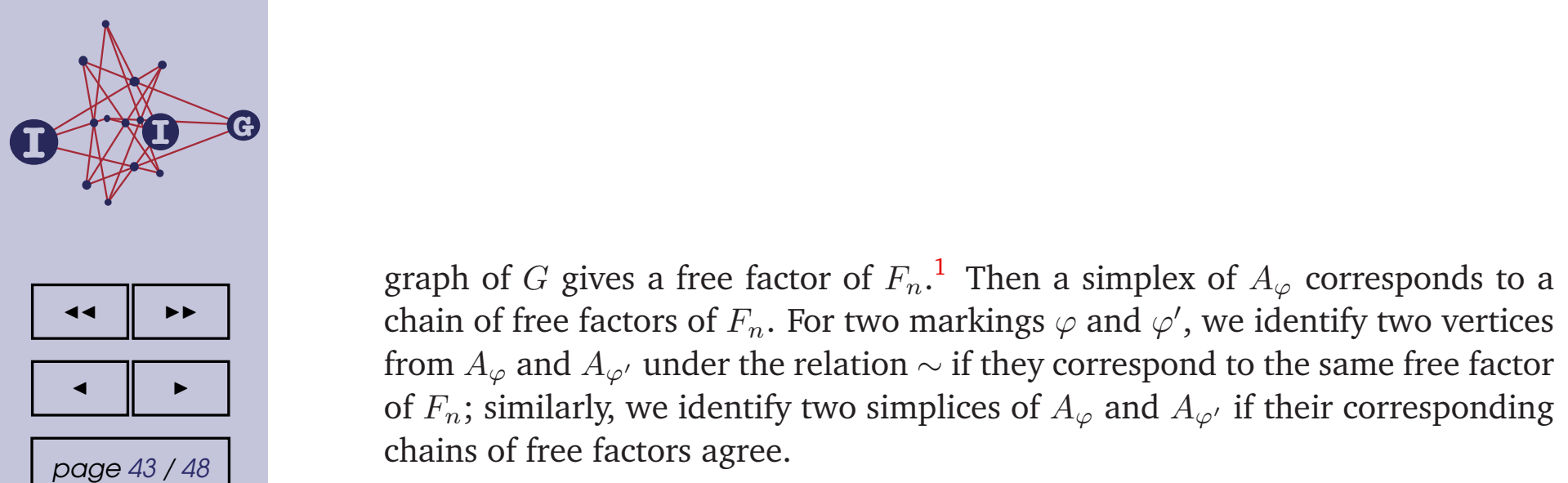

graph of $G$ gives a free factor of $F_{n}$. chain of free factors of $F_{n}$. For two markings $\varphi$ and $\varphi^{\prime}$, we identify two vertices from $A_{\varphi}$ and $A_{\varphi^{\prime}}$ under the relation $\sim$ if they correspond to the same free factor chains of free factors agree.

Remark 10.1. A more geometric way to define and understand the equivalence relation $\sim$ in defining $\mathcal{C G}\left(F_{n}\right)$ in equation (12) is as follows. Given a marked graph $\varphi: R_{n} \rightarrow G$, every core subgraph of $G$ is pulled back to a subgraph of $R_{n}$. Similarly a chain of core subgraphs of $G$ is pulled back under $\varphi$ to a chain of subgraphs of $R_{n}$. Given two marked graphs $\varphi_{1}: R_{n} \rightarrow G_{1}$ and $\varphi_{2}: R_{n} \rightarrow G_{2}$, and two chains of core subgraphs in $G_{1}$ and $G_{2}$ respectively, then the corresponding simplices in $A_{\varphi_{1}}$ and $A_{\varphi_{2}}$ are defined to be equivalent if their pull-backs in $R_{n}$ under $\varphi_{1}$ and $\varphi_{2}$ are homotopy equivalent chains of subgraphs.

This is closely related to the definition of the curve complex $\mathcal{C}(S)$, where a simplex is defined to a collection of homotopy classes of disjoint simple closed curves. It is worthwhile to point out that in the markings for the Teichmüller space $T_{g}, \varphi: S \rightarrow \Sigma$, the surfaces $S$ and $\Sigma$ are homeomorphic, and we can consider simple closed curves on a common surface. On the other hand, for marked graphs, $G$ is usually not homeomorphic to $R_{n}$. This is the reason why we need to pull back chains of core subgraphs of $G_{1}, G_{2}$ to $R_{n}$ and require them to be homotopic.

Since every free factor of $F_{n}$ and every chain of free factors arise from core subgraphs this way from a core subgraph and a chain of core subgraphs, it follows that the core graph complex $\mathcal{C G}\left(F_{n}\right)$ is isomorphic to the factor complex in [22].

Remark 10.2. For each marked graph $\varphi: R_{n} \rightarrow G$ with only nodes of valency 3 , the finite simplicial complex $A_{\varphi}$ seems to be an analogue of an apartment of a spherical Tits building. One reason for this is that the spherical Tits building can also be obtained from the apartments by identifying along simplices of smaller dimension. As a consequence, the spherical Tits building has the same dimension as the apartments. Clearly, $\mathcal{C G}\left(F_{n}\right)$ also has the same dimension as $A_{\varphi}$.

Now we use the simplices in $\mathcal{C G}\left(F_{n}\right)$ to decompose the boundary $\partial X_{n}(\varepsilon)$ into boundary components. For a simplex $\sigma \in \mathcal{C G}\left(F_{n}\right)$, consider all subcomplexes $A_{\varphi}$ that contain $\sigma$. We pointed out earlier that the faces of the polytope $\Sigma_{\varphi}(\varepsilon)$

\footnotetext{
${ }^{1}$ Geometrically, we can visualize a rose $R_{n}$ obtained from $G$ by collapsing some edges so that a core subgraph $C$ of $G$ is mapped to the union of $r(C)$ petals (each loop in $C$ is mapped to one petal). Note that the petals obtained in this way are usually different from the petals in $R_{n}$ under the marking $\varphi: R_{n} \rightarrow G$. Therefore, the marking $\varphi$ plays a crucial role here.
} 

[12] _ Cohomologie d'immeubles et de groupes $S$-arithmétiques, Topology 15 (1976), 211-232.

[13] M. R. Bridson and A. Haefliger, Metric Spaces of Non-Positive Curvature, Grundlehren Math. Wiss. 319, Springer-Verlag, Berlin, 1999.

[14] K. Brown, Buildings, Springer Monogr. Math.. Springer-Verlag, 1998.

[15] _ Cohomology of Groups, Corrected reprint of the 1982 original. Grad. Texts in Math. 87, Springer-Verlag, 1994.

[16] M. Culler, Finite groups of outer automorphisms of a free group, in Contributions to group theory, pp. 197-207, Contemp. Math., vol. 33, Amer. Math. Soc., 1984.

[17] M. Culler and K. Vogtmann, Moduli of graphs and automorphisms of free groups, Invent. Math. 84 (1986), 91-119.

[18] Y. Guivarch, L. Ji and T. C. Taylor, Compactifications of Symmetric Spaces, Progr. Math. 156, Birkhäuser Boston, 1998.

[19] U. Hamenstädt, Geometry of the mapping class groups III: Quasiisometric rigidity, preprint. arXiv: math/0512429.

[20] J. Harer, The cohomology of the moduli space of curves, in Theory of moduli, pp. 138-221, Lecture Notes in Math. vol. 1337, Springer, 1988.

[21] W. Harvey, Boundary structure of the modular group, in Riemann surfaces and related topics, pp. 245-251, Ann. of Math. Stud. 97, Princeton Univ. Press, 1981.

[22] A. Hatcher and K. Vogtmann, The complex of free factors of a free group, Quart. J. Math. Oxford Ser. 49 (1998), 459-468.

[23] N. Ivanov, Mapping Class Groups, in Handbook of geometric topology, pp. 523-633, North-Holland, 2002.

[24] N. Ivanov and L. Ji, Infinite topology of curve complex and nonPoincaré duality of Teichmüller modular groups, Enseign. Math. 54 (2008), 381-395.

[25] L. Ji, Buildings and their applications in geometry and topology, Asian J. Math. 10 (2006), 11-80.

[26] _ Integral Novikov conjectures and arithmetic groups containing torsion elements, Commun. Anal. Geom. 15 (2007), 509-533. 

Arithmetic Groups and Their Generalizations: What, Why and

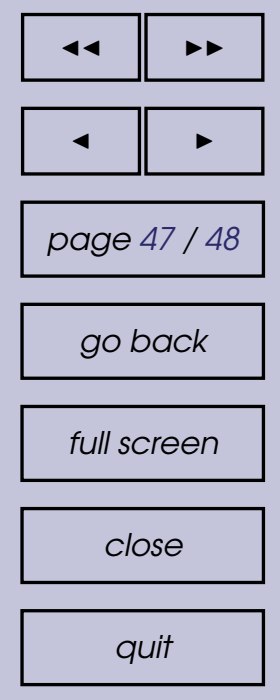
How, AMS/IP Stud. Adv. Math. 43, Amer. Math. Soc., Providence, RI; Intern. Press, Cambridge, MA, 2008.

[28] , A summary of some work of Gregory Margulis, Pure Appl. Math. Q. 4 (2008), 1-69.

[29] — The integral Novikov conjectures for linear groups containing torsion elements, J. Topol. 1 (2008), 306-316.

[30] _ Steinberg representations, and duality properties of arithmetic groups, mapping class groups and outer automorphism groups of free groups, in Representation Theory, pp. 71-98, Contemp. Math., vol. 478, Amer. Math. Soc., Providence, RI, 2009.

[31] L. Ji and S. Wolpert, A cofinite universal space for proper actions for mapping class groups, in In the Tradition of Ahlfors-Bers, V, eds. M. Bonk, J. Gilman, H. Masur, Y. Minsky and M. Wolf, Contemp. Math., vol. 510, pp. 151-163, Amer. Math. Soc., 2010.

[32] B. Kleiner and B. Leeb, Rigidity of quasi-isometries for symmetric spaces and Euclidean buildings, Publ. Math. Inst. Hautes Études Sci. 86 (1997), 115-197.

[33] S. Krstic and K. Vogtmann, Equivariant outer space and automorphisms of free-by-finite groups, Comment. Math. Helv. 68 (1993), 216262.

[34] B. Leeb, A Characterization of Irreducible Symmetric Spaces and Euclidean Buildings of Higher Rank by Their Asymptotic Geometry, Bonner Math. Schriften 326, Universität Bonn, Mathematisches Institut, Bonn, 2000.

[35] E. Leuzinger, An exhaustion of locally symmetric spaces by compact submanifolds with corners, Invent. Math. 121 (1995), 389-410.

[36] G. Mostow, Strong rigidity of locally symmetric spaces, Annals of Math. Stud., No. 78, Princeton University Press, 1973.

[37] G. Mostow and T. Tamagawa, On the compactness of arithmetically defined homogeneous spaces, Ann. of Math. 76 (1962), 446-463.

[38] D. Mumford, A remark on Mahler's compactness theorem, Proc. Amer. Math. Soc. 28 (1971), 289-294. 
